\title{
El nuevo Plan Hidrológico de la Demarcación del Guadalquivir: avances y dificultades en el proceso de cambio de modelo de gestión del agua
}

\author{
Leandro del Moral Ituarte*
}

\section{Resumen}

Tras un proceso de seis años en aplicación de la Directiva Marco del Agua, la Confederación Hidrográfica del Guadalquivir presentó en enero de 2011 el proyecto de plan hidrológico para esta demarcación. Pese a los innegables avances que este documento supone, en el artículo se analizan las carencias fundamentales que presenta en relación con los nuevos objetivos de calidad, buen estado, riguroso análisis económico, así como transparencia y participación pública activa que el nuevo marco institucional exige.

\section{Palabras clave}

Directiva Marco del Agua; Plan Hidrológico; Guadalquivir; Gestión del agua

\section{Abstract: The new Water Management Plan of the Guadalquivir river: progress and difficulties in changing the water management model}

After implementing the Water Framework Directive for six years, the Guadalquivir River Basin Authority submitted its draft water management plan in January 2011. It is undeniable that this document represents progress. Yet, this paper analyzes the main shortcomings of the Plan with regard to the new objectives pertaining to quality, good condition, thorough economic analysis and transparency and active public participation required by the new institutional framework.

\section{Key words}

Water Framework Directive; Water Managemen; Plan; Guadalquivir river; Water management 
El actual proceso de planificación de cuencas inspirado por la Directiva Marco del Agua (DMA) está significando indudables avances. En el caso del Guadalquivir, entre estos avances destacan el incremento del conocimiento de la cuenca, la comprensión y aceptación por parte del equipo redactor de las tendencias de reducción de las demandas urbanas de agua, la confirmación de la necesidad de frenar la expansión del regadío o el intento de fijar dotaciones más eficientes de riego. También es justo reconocer el esfuerzo que el proceso ha requerido por parte de los técnicos de la Oficina de Planificación, así como su disponibilidad a debatir abiertamente, como se puso de manifiesto en las Jornadas organizadas por el Observatorio de la Directiva Marco del Agua en Sevilla los días 13 y 14 de mayo 2011. Sin embargo, a la vista del contenido de la Propuesta de Proyecto de Plan Hidrológico de la Demarcación Hidrográfica del Guadalquivir (PHDHG, 2010), las grandes posibilidades que la aplicación de la DMA ofrece para la reorientación de la planificación y gestión del agua siguen sin ser aprovechadas en esta Demarcación.

Las principales conclusiones derivadas del análisis del proyecto del PHDHG son las siguientes:

\section{Desgobierno territorial y expansión del regadío}

El problema clave, aunque evidentemente no único, de la cuenca del Guadalquivir es la expansión descontrolada de la superficie de regadío a lo largo de los últimos 25 años. En cada nuevo documento que se presenta la superficie de regadío se ve incrementada en decenas de miles de hectáreas: el documento Documentación Básica del Plan Hidrológico de cuenca (1988) la estimaba en 418.069 ha; el propio Plan Hidrológico todavía vigente, aprobado en 1998 aunque utilizando cifras de 1992, la cifraba en 443.024 ha; el Informe de los artículos 5 y 6 (marzo 2005, pág.
33) seguía manteniendo esta misma cifra ${ }^{1}$; el Esquema de Temas Importantes (ETI, julio 2008, pág. 41) la cifraba en 801.157 ha (con datos de diciembre de 2007) ${ }^{2}$; el actual proyecto de PHDHG, en 845.986 ha. ${ }^{3}$ La demanda de agua para riego alcanza un $87 \%$ del total de las demandas de la cuenca, 3.329,5 hm3 (el ETI lo cifraba en $3.485 \mathrm{hm} 3 / \mathrm{año}$ ), de un total de 3.833,2 hm3 de demanda en los diferentes usos.

El Guadalquivir es rehén del regadío: este es el hecho principal y clave que condiciona la gran dificultad para avanzar hacia los objetivos de la Directiva Marco, es decir, conservar o restaurar el buen estado ecológico de las aguas.

Este tema debería recibir una atención específica en el Plan: los agentes sociales sin ánimo de lucro que defienden los intereses generales de la sociedad lo vienen pidiendo en todas sus alegaciones, desde el debate sobre el diagnóstico de la cuenca (Informe de los artículos 5 y 6, 2005) hasta el actual proyecto de Plan, pasando por las alegaciones al citado ETI de 2008. No basta con informar que el valor marginal del agua en el olivar es alto $(0,96 € / \mathrm{m} 3)$ y asumir ese dato como una variable independiente, incuestionable y suficiente. Esa actitud nos sitúa precisamente en el terreno de la vieja política hidráulica de oferta y de justificación de la irresponsabilidad frente a las inercias.

\footnotetext{
1 Es significativa la consideración que este Informe presenta sobre la evolución de las extracciones de aguas subterráneas : «En cuanto a la evolución temporal de la demanda, en los últimos seis meses que se lleva elaborando este balance los recursos comprometidos han aumentado en $45 \mathrm{Hm} 3$ dentro de los perímetros de las masas de agua. Considerando este periodo como normal obtendríamos un incremento medio de 90 Hm3 anuales" (Informe de los artículos 5 y 6, marzo 2005 , 133).

2 Este mismo documento (ETI, borrados julio 2007, documento definitivo octubre 2010) señala que en 1980 la superficie de regadío en la cuenca era 317.138 ha.

3 "Lo más destacable en la evolución de la distribución de cultivos es el fuerte crecimiento del olivar de regadío, que pasa de 74.066 ha en 1989 a 467.588 ha en 2008 , creciendo a un ritmo medio anual del $13 \%$ en el primer periodo, lo que supone unas 214.000 nuevas hectáreas de olivar entre 1989 y 1999, e incrementándose en otras 179.000 hectáreas entre 1999 y 2008 (tasa de crecimiento anual del 5\%)" (Memoria de la Propuesta de Proyecto de PHDHG 2010, 84).
} 
Hace falta analizar, aprender y diseñar medidas para afrontar los factores, complejos y profundamente enraizados, que han permitido este fenómeno: dejación de autoridad, tolerancia social hacia la ilegalidad en el uso del agua, unida a desinformación sobre sus nefastas consecuencias ambientales y económicas, falta de medios de la administración, redes de influencia, connivencia, mecanismos de presión de los agentes beneficiados y, en su caso, corrupción. Si estos factores no se rectifican, la dinámica de expansión se mantendrá en el futuro. Es necesario incorporar al Plan la consideración expresa y rigurosa del problema de la mala gobernanza del agua (indisciplina y descontrol en la administración del dominio público hidráulico), incluyendo todos sus factores explicativos (políticos, sociales, económi$\cos$ y administrativos), sus consecuencias y las medidas y alternativas propuestas para su resolución.

Los técnicos de la Oficina de Planificación -por no decir las consultorías contratadas- no deben asumir como una responsabilidad propia esta realidad y sus implicaciones para el diagnóstico, objetivos posibles y medidas alternativas, respondiendo a la defensiva a las críticas de los agentes sin ánimo de lucro. Esta dinámica de desgobierno constituye una condición de entorno de todo el proceso planificador que no debe distorsionar -como creemos que lo hace- el rigor y la solvencia de los trabajos de los que son responsables. Se trata de una decisión fundamental sobre el modelo de desarrollo territorial que condiciona la gestión de la cuenca cuya responsabilidad debe ser asumida explícitamente ya no por el Presidente de la CHG sino por el conjunto del Comité de Autoridades Competentes.

\section{Inadecuada valoración del nivel de recuperación de costes}

El borrador de Plan consolida el discurso de que los costes de los servicios de agua ya se están recuperando en un porcentaje muy elevado, afirmándose, contra toda evidencia, que los ingresos obtenidos por los organismos que prestan los servicios significan el $85 \%$ de los costes. Este discurso se apoya en la sobreestimación de los índices de recuperación de estos costes, construida mediante la eliminación o minoración de numerosas partidas de coste, que por diversos motivos se consideran no repercutibles, además del cómputo inadecuado de costes ambientales. Esta recuperación de costes insuficiente compromete tanto el alcance de los objetivos de buen estado como la viabilidad económica del Plan y la sostenibilidad financiera de la administración del agua.

El tratamiento de este tema refuerza la principal debilidad del PHDHG propuesto: el desconocimiento de la realidad de los problemas (o quizás el esfuerzo por ocultarlos). En los debates públicos y en la documentación técnica públicamente disponible existen datos y argumentaciones que avalan el hecho de que la recuperación de los costes se sitúa en torno al $23 \%$, incluso en ausencia de una estimación rigurosa de los costes ambientales de los servicios del agua.

Tampoco hay que ignorar que los usuarios urbanos soportan un nivel sensiblemente más elevado de recuperación de costes, tanto por los mayores niveles de cánones y tarifas como por la internalización -principalmente en los costes de potabilización y de depuración- de impactos en la calidad del agua ocasionados por el regadío. A través de este hecho se produce una subvención cruzada entre diferentes sectores de uso del agua. 


\section{Falta de rigor en la definición de escenarios para masas de agua superficiales}

La situación real de las masas de agua de la categoría ríos y aguas de transición -de manera especial, su mal estado en lo que se refiere a los aspectos hidromorfológicos y biológicos, especialmente comunidades de peces- hace poco plausible el logro de los objetivos ambientales establecidos. Los resultados de los estudios hechos recientemente indican el paupérrimo estado de conservación que presenta la comunidad de peces autóctonos de la cuenca del Guadalquivir, y el grave estado físico, químico y biológico en el que se encuentra el estuario, lo que refleja un serio y generalizado deterioro de los ecosistemas fluviales y estuarios.

Es muy poco verosímil que el $91 \%$ de las masas de agua categoría ríos (356 de un total de 392) y el $100 \%$ de las de transición vayan a alcanzar el buen estado o el buen potencial en el año 2015, como asegura la propuesta de PHDHG. Este dato suscita enormes dudas sobre el rigor y la solvencia de los análisis realizados. Estas previsiones contrastan con los objetivos de los planes hidrológicos de Holanda o Francia, en donde el objetivo a alcanzar es el $20 \%$ y el $60 \%$ respectivamente de buen estado en 2015. ¿Es que la situación del maltrecho Guadalquivir es mejor que la de los ríos franceses?

\section{Debilidades en el tratamiento de las masas de agua subterráneas}

En las condiciones climáticas e hidrológicas propias de la cuenca del Guadalquivir, las aguas subterráneas son vitales para el mantenimiento del caudal estival de las masas de agua superficiales. En la propuesta de PHDG se habla poco de uso conjunto y de recarga de acuíferos y se presta poca atención a los nacimientos de los ríos. Se diferencia sistemáticamente en mapas y tablas las masas de aguas superficiales de las subterráneas, perdiéndose la perspectiva de fusión final de ambas en una misma agua.

La propuesta de PHDG no da la suficiente importancia a las aguas subterráneas situadas en materiales de baja permeabilidad, que ocupan del orden del $50 \%$ de la superficie de la cuenca y en la que sólo se admite una explotación del orden de $70 \mathrm{hm}$ 3/año. En realidad se trata de territorios de una alta tasa de perforaciones ilegales, sin apenas estudios ni controles, en los que las extracciones son muy superiores y afectan tanto a ríos y arroyos como a masas de aguas subterráneas limítrofes.

El indicador de estado cuantitativo en base a la relación extracciones/recursos presenta numerosas incertidumbres. Su valor tampoco refleja necesariamente la buena o mala calidad de los ecosistemas asociados. El estado cuantitativo debería medirse prioritariamente por la evolución en continuo de caudales surgentes y niveles piezométricos, cuyo uso está perfectamente en sintonía con la DMA, que prevé el control del estado cuantitativo a través de redes de seguimiento.

\section{Carencias de coordinación interadmin- istrativa e integración}

Los diagnósticos, objetivos y medidas presentados en el análisis concreto de las masas de agua (tramos de río, acuíferos, lagos o sectores costeros, a escala de detalle, como establece la DMA), ponen de manifiesto un elevado desconocimiento o falta de atención a las actuaciones, proyectos o planes que realmente han incidido recientemente, están incidiendo o está previsto que incidan en los territorios concretos. En este nivel de escala de detalle se ponen de manifiesto de manera especialmente acusada los déficits de información y de participación pública, así como la carencia, en muchas ocasiones, de elementales procesos de coordinación interadministrativa. Lo 
hemos comprobado concreta y detalladamente en aquellos casos en los que la demanda social ha solicitado el asesoramiento del Observatorio y en donde los propios agentes sociales locales han aportado información: estuario del Guadalquivir, río Guadaíra, río Castril, acuíferos de BedmarJódar, Mancha Real-Pegalajar, Caniles-Baza, etc. Todo ello refleja las dificultades que presenta abordar la planificación de sistemas complejos con un gabinete que centraliza las decisiones, mientras que los trabajos de recopilación, tratamiento y evaluación de la información se encargan a empresas consultoras externas. En contraposición a este modelo, en las Jornadas del ODMA se debatió sobre las nuevas tendencias para abordar estos problemas en diferentes ámbitos; la gestión del conocimiento, la organización de la inteligencia colectiva, la creación de redes sociales organizadas a través de las Tecnologías de la Información (TI), etc.

\section{Cuantificación de recursos naturales y cambio climático}

El problema se agrava a la vista de la reevaluación de los recursos naturales de la cuenca, con el telón de fondo del cambio climático, que por fin -no como en las fases anteriores: Informe de los artículos 5 y 6 y ETI- la propuesta de PHDG realiza cumpliendo el mandato de la Instrucción de Planificación Hidrológica. De los datos presentados se desprende una reducción de entre el 10\% y el $20 \%$ de las aportaciones naturales, según el sistema de explotación de que se trate, en la serie reciente (1980-2005) respecto de la larga (19402005) (PHDG, Memoria y Anejo $n^{\circ} 2$ Inventario de recursos hídricos).

\begin{tabular}{|c|c|c|c|}
\hline \multicolumn{5}{|c|}{ Serie de Aportaciones (Hm3/año) } & Diferencia \\
\hline & $1980 / 82-2005 / 2006$ & $1940 / 41-2005 / 2006$ & $1.289(18,3 \%)$ \\
\hline Media & 5.754 & 7.043 & $1.227(24,16 \%)$ \\
\hline Mediana & 3.851 & 5.078 & \\
\hline
\end{tabular}

Figura 1. Recursos hídricos disponibles en la DHG. Fuente: Basado en la tabla 18. Memoria P,H. de Guadalquivir.

En cualquier caso, se sigue sin extraer de manera clara y explícita las implicaciones actuales y para el futuro de esta reevaluación de los recursos disponibles. En la simulación del impacto futuro de cambio climático se aplica sin concreción territorial la cifra del $8 \%$ de disminución de recursos que con carácter general indica el Reglamento de la
Planificación (basada en las conclusiones del estudio del Centro de Estudios Hidrográficos del CEDEX sobre la evaluación de los efectos del cambio climático en los recursos hídricos), sin definirse con claridad a cuál de las dos cifras (serie reciente o larga) se aplica.

\begin{tabular}{|c|c|c|c|}
\hline \multirow{2}{*}{ Cuenca } & \multicolumn{3}{|c|}{ Brecha (Hm3/año) } \\
\cline { 2 - 4 } & Sin cambio de climático & Con cambio climático & 15 \\
\hline Demandas Aguas Reguladas & 287 & 330 & 20 \\
\hline Demanda Aguas No Reguladas & 124 & 153 & \\
\hline
\end{tabular}

Figura 2. Efectos del cambio climático. Fuente: Memoria P.H. de la DHG. Tabla 87.

Queda pendiente la necesidad de incorporar la consideración del cambio climático en la definición de los objetivos, programa de medidas, seguimien- to y control de la planificación hidrológica, que debe realizarse teniendo en cuenta tanto los impactos generados por las presiones en el estado 
actual, como los impactos que generará el cambio climático en próximas décadas. Estas nuevas situaciones exigirán una revisión de las condiciones de referencia utilizadas hasta la fecha y la incorporación de mecanismos que permitan la revisión periódica de los efectos de las medidas en relación con el cambio climático.

\section{Régimen de caudales ecológicos: profundamente inadecuados y no concertados}

Las comprobaciones realizadas, tanto de los caudales mínimos de todo el año y máximos estivales, como del conjunto de valores que componen los regímenes propuestos, muestran que en general, comparados con los caudales medios y mínimos mensuales, son muy bajos, no siguen el rango natural de variabilidad y resultan insuficientes para obtener un comportamiento hidrológico en el sistema que asegure su conservación. Los caudales ambientales mínimos se reducen, en general, a la mitad de los fijados por el Plan Hidrológico vigente (1998), con la justificación de que aquellos estaban concebidos como caudales de dilución, que con la mejora de los niveles de depuración de la cuenca dejarían de ser necesarios. En muchas ocasiones, el régimen propuesto produce un hábitat insuficiente para el desarrollo de la vida piscícola, al proporcionar valores inferiores al $50 \%$ del hábitat que se puede alcanzar en los tramos. Las variaciones intraanuales que se proponen tampoco van a permitir que se cumplan los procesos naturales atribuibles a los cambios estacionales de los caudales, minimizando o impidiendo que se puedan mantener en unos valores aceptables las componentes, funcionalidad y complejidad del sistema fluvial que se trata de preservar con la aplicación de estos caudales.

Es necesaria una revisión en profundidad tanto del proceso, como de los trabajos técnicos, metodologías (incluida la extrapolación de resultados) y selección de los resultados incluidos en el Anexo 5 del borrador del PHDG, para tratar de corregir las graves deficiencias que contiene el documento. Esta revisión es necesaria para obtener una propuesta de regímenes ecológicos que realmente contribuya a alcanzar y mantener el buen estado ecológico de las masas de agua en los tramos en los que se implementen, tal como propone la DMA.

En el apartado 8 (pág. 34-35) del Anejo no 5.Implantación del régimen de caudales se dice:

"La implantación de los caudales ecológicos debe desarrollarse en cada caso conforme a un proceso específico de concertación, para conciliar los requerimientos ambientales (...) con los usos actuales dentro de cada masa de agua, manifestados en los correspondientes títulos habilitantes (...) Como parte del desarrollo de las tareas de los respectivos contratos, las tres consultoras encargadas de realizar los estudios técnicos elaboraron diversos documentos en los que se plantean de forma teórica las diferentes aproximaciones posibles para realizar la concertación (...) Estas aproximaciones planteadas en los citados documentos constituyen un punto de partida, aunque su carácter eminentemente teórico conlleva la necesidad de detallar y concretar los procesos de concertación, tarea que necesariamente requiere de una implicación importante de las respectivas Oficinas de Planificación Hidrológica. Asimismo, de forma coordinada con la $\mathrm{OPH}$ se propone desarrollar el proceso de concertación en las 32 masas que han sido objeto de estudios específicos. Una vez finalizado el proceso de concertación, el cual tendrá lugar durante el período de consulta pública del borrador del Plan Hidrológico de cuenca, se plantearán tres situaciones (...)".

En el apartado 9 (pág. 36) de este mismo Anejo ${ }^{\circ}$ 5 se señala:

"La presentación de los resultados de la determinación del régimen de caudales ecológicos se desarrolla en dos momentos diferentes: Una primera fase corresponde a los resultados provenientes de los estudios técnicos, previamente al proceso de concertación descrito en el apartado 8, y que son incluidos en el borrador de Plan Hidrológico de cuenca, el cual va a ser sometido a consulta pública. 
Una segunda fase tendrá lugar una vez concluidos tanto el proceso de concertación de los regímenes de caudales ecológicos como la consulta pública del borrador de Plan"

Sin embargo, durante el periodo de consulta pública de la propuesta de PHDG no se ha previsto ningún proceso público de concertación (información, consulta pública y participación activa, como establece el art. 3.4.6. de la Instrucción del Planificación Hidrológica, IPH) sobre el régimen de caudales ambientales, cuyas características se han desconocido hasta la publicación de esta propuesta.

El artículo 3.4.6 de la IPH (Proceso de concertación del régimen de caudales) establece que:

"En este último nivel se incluirá una fase de negociación o resolución de alternativas, donde estén representados adecuadamente todos los actores afectados: organismos oficiales, usuarios, organizaciones económicas sociales y ambientales, expertos y en el caso concreto de los usos energéticos, organismos oficiales responsables del suministro eléctrico. Este proceso deberá ser previo a la inclusión del régimen de caudales en el plan hidrológico".

El hecho de que la concertación se realice una vez elaborado el Plan pone en riesgo el mismo, pues teniendo en cuenta el estado de las masas de agua de la cuenca, la definición de los regímenes de caudales necesarios para alcanzar su buen estado va a condicionar o limitar, en cantidad y distribución temporal, el agua disponible para los diferentes usos en la cuenca, definidos en el Plan. La conclusión fundamental es que en el Guadalquivir la presión de las demandas agrarias -y en menos medida energéticas- y la capacidad de condicionar las decisiones por parte de los representantes de estos sectores dificulta gravemente fijar regímenes de caudales que cubran las necesidades ecológicas mínimas de las masas de agua y condiciona la aplicación de los procedimientos legalmente establecidos para su concertación.

\section{Falta de atención al aumento de la vulnerabilidad frente a la inundación: consecuencia de la desconexión de la gestión del agua de la ordenación urbanística y territorial.}

El déficit de gobierno del territorio, en este caso urbanístico, está en la base de otro fenómeno de creciente impacto al que no se presta la debida atención en el Plan: las inundaciones, derivadas de un aumento de la vulnerabilidad. En este sentido la tradicional y todavía creciente ocupación desordenada de llanuras de inundación sigue requiriendo una atención e intervención mayor por parte del organismo de cuenca. Por otra parte, cada vez se dejan sentir con más intensidad las consecuencias del sellado e impermeabilización de suelos, con impacto en procesos de hidrología urbana que crean nuevas situaciones de riesgo o agravan las existentes.

Una de las consecuencias más inadvertidas hasta el momento que la actividad urbanística está provocando es la alteración del funcionamiento hidrológico de espacios cada vez más extensos, con resultados de intensificación de la incisión y deterioro de la red de drenaje. La invasión o eliminación de cauces y las grandes superficies de suelo impermeabilizados propiciado por desarrollos urbanísticos no supervisado, como debiera, por la administración de aguas, están alterado sensiblemente en las aglomeraciones urbanas la manera en que fluyen las aguas superficiales y la infiltración a las aguas subterráneas. Un mayor volumen de agua concentrado en menos tiempo llega por superficie o a través de canalizaciones y de colectores a la red de drenaje. La reducción del espacio de los ríos como consecuencia de los rectificados, canalizaciones, cambios de trazado, ocupaciones del cauce y sus riberas, etc., imposibilitan los procesos naturales de laminación y disipación de energía. Todo ello, está provocando profundas incisiones en el suelo, el desmoro- 
namiento y desplome de las riberas, el deterioro de los ecosistemas fluviales y el agravamiento del riesgo de inundación.

Para paliar estos problemas se debe incluir en el PHDG la consideración de medidas correctoras del sellado de los suelos que reduzcan las escorrentías y faciliten la retención y la infiltración. Es decir, sistemas urbanos de drenaje sostenibles, que incluyen potenciación de suelos permeables, franjas filtrantes, pozos y zanjas de infiltración, drenes filtrantes, cunetas verdes, depósitos de infiltración, depósitos superficiales de detención, red de recogida y tratamiento de aguas pluviales separada de la red de saneamiento, depósitos enterrados de detención (depósitos de tormentas), estanques de retención, humedales artificiales, incentivos a la construcción de aljibes privados, cubiertas vegetales, etc.

El Plan debe entrar en estos temas, incorporando a la su normativa las determinaciones de los instrumentos de Ordenación Territorial (Plan de Ordenación del Territorio de Andalucía, POTA, en las zonas que sean de aplicación, y planes subregionales), respecto a la red de drenaje (especialmente el artículo 90 del POTA). EI PHDG debe incorporar en su normativa el contenido del Informe al que se hace referencia en el artículo 25.4 del Real Decreto Legislativo 1/2001, de 20 de julio, por el que se aprueba el texto refundido de la Ley de Aguas (TRLA), modificado por la Disposición final primera de la Ley $11 / 2005$, de 22 de junio, informe también regulado por el artículo 42 de la Ley de Aguas de Andalucía. Esto contribuiría a mejorar la necesaria coordinación con la normativa de ordenación territorial y urbanística relativa a la red hidrológica, como establecen el artículo 41.1 del TRLA y la Instrucción de Planificación Hidrológica (artículo 8. Programas de medidas, 8.1. Procedimiento de análisis $y$ definición del programa).

\section{Ausencia de impulso institucional a la participación activa sobre la propuesta concreta de PHDHG}

Tras un acto público de presentación de la propuesta de PHDHG celebrado el 24 de febrero en Sevilla, la Confederación Hidrográfica del Guadalquivir (CGH) dio por finalizado el proceso de participación pública en su elaboración. A este acto añadió en el mes siguiente una presentación de media jornada sobre caudales ambientales de carácter informativo en Córdoba.

A partir de ahora, la CHG recibirá las alegaciones que se presenten por escrito, a la vez que se establecerán contactos directos entre la $\mathrm{CHG}$ y los agentes que lo soliciten. Esto implica que la CHG no impulsará el debate público sobre los contenidos concretos del Plan: en todo el proceso anterior los usuarios y el público en general no han tenido conocimiento de los objetivos, las excepciones y las medidas concretas (plagadas de errores, como hemos señalado) propuestas para cada masa de agua de la Demarcación, que sólo se han conocido tras la publicación del proyecto en diciembre pasado. El único acto de participación pública activa que se ha desarrollado ha sido el impulsado por el ODMA de la FNCA en el que se contó con la presencia y activa participación, muy valiosa, de responsables y técnicos de la CHG y de las Consejerías de Medio Ambiente y Agricultura de la Junta de Andalucía.

\section{Las claves de política económica, social y territorial que subyace a los grandes problemas del PHDHG}

La conclusión fundamental es que, al margen de la nueva lógica, metodología y terminología de la Directiva Marco, que apunta a la mejora del estado ecológico en la escala de masa de agua (sin perder la dimensión de cuenca y demarcación), el núcleo de la propuesta de Plan del Guadalquivir gira en torno a la reducción de la "brecha" entre recursos disponibles y demandas, el antiguo 
"déficit estructural", a escala de sistema de explotación. Esta brecha es el resultado del proceso político (de política de aguas, de política territorial) de fondo: el incremento de las demandas que no se ha querido o podido, ni se sigue queriendo o pudiendo, evitar. Este es el núcleo central del problema del agua en la cuenca, problema grave y complejo, que se ha evitado poner a debate público.

Pese a todo, los grupos de presión agrarios no se dan por satisfechos: ya han iniciado una campaña denuncia de las dotaciones de riego por cultivo establecidas en la propuesta de PHDHG (reducidas respecto de las vigentes), defendiendo una dinámica de presiones que agravará la tendencia al colapso ecológico que amenaza seriamente a la cuenca, como se ha puesto de manifiesto en el estuario en los últimos años.

En palabras del Dictamen de la Comisión Científica para el Estudio de las Afecciones del Dragado del Río Guadalquivir (noviembre 2010):

"La situación actual requiere la intervención rápida y coordinada de las administraciones implicadas en la planificación y gestión del estuario, de la cuenca del Guadalquivir y de la costa, de modo que se revierta la tendencia actual que lleva al colapso al estuario y a la costa que de él depende, y por tanto al Parque Nacional de Doñana, cuyas marismas forman parte del propio estuario".

\section{Referencias}

Comisión Científica para el estudio de las afecciones de las obras de dragado del río Guadalquivir. Dictamen de la Comisión Científica para el Estudio de las Afecciones del Dragado del Río Guadalquivir, 2010. <http://www.marm.es/es/calidad-y-evaluacionambiental/temas/evaluacionambiental/dictamen_cc_drag_guadalquivir_tcm7147410.pdf> (Consulta el 30 de julio 2011).

Confederación Hidrográfica del Guadalquivir. Esquema de Temas Importantes, 2010. <http://www.chguadalquivir.es/export/sites/default/portalchg
/planHidrologicoDemarcacion/participacionPublica/consulta Publica/ficheros/Esquema_Temas_Importantes.pdf>

(Consultado el 30 julio 2011).

Confederación Hidrográfica del Guadalquivir. Propuesta de Proyecto de Plan Hidrológico de la Demarcación Hidrográfica del Guadalquivir, 2010.

$<$ <ttp://www.chguadalquivir.es/opencms/portalchg/planHidr ologicoDemarca-

cion/participacionPublica/consultaPublica/\#apartado4>

(Consultado el 30 julio 2011).

Directiva 2000/60/CE del Parlamento Europeo y del Consejo, de 23 de octubre de 2000, por la que se establece un marco comunitario de actuación en el ámbito de la política de aguas.

Ministerio de Medio Ambiente y Medio Rural y Marino (MARM), Guía técnica para la caracterización de medidas, versión 3, mayo de 2009.

ORDEN ARM/2656/2008, de 10 de septiembre, por la que se aprueba la instrucción de planificación hidrológica.

Texto Refundido de la Ley de Aguas (TRLA), compuesto por el Real Decreto Legislativo (RDL) 10/2001, de 5 de julio, y sus sucesivas modificaciones, entre las cuales cabe destacar la Ley 24/2001, de 27 de diciembre, la Ley 62/2003, de 30 de diciembre, la Ley 11/2005, de 12 de junio, y el Real Decreto Ley 4/2007, de 13 de abril.

Real Decreto 907/2007, de 6 de julio, por el que se aprueba el Reglamento de la Planificación Hidrológica.

<www.boe.es/boe/dias/2007/07/07/pdfs/A2936 1-29398.pdf> 


\section{Cita del artículo}

DEL MORAL ITUARTE, Leandro. El nuevo Plan Hidrológico de la Demarcación del Guadalquivir: avan-ces y dificultades en el proceso de cambio de modelo de gestión del agua. Hábitat y Sociedad, 2011, no 2, p. 187-196.

$<$ www.habitatysociedad.us.es>.

http://dx.doi.org/10.12795/HabitatySociedad.2011.i2.10 